\title{
Breastfeeding After Breast Augmentation
}

\author{
E. Charles R. Lee, David Topchian, and Jane Petro
}

\subsection{Submitted by Lee: April 17,2008}

What is the general consensus on patients with silicone gel implants. Can they breastfeed? I have a patient who had breast augmentation 2 years ago with textured gel implants placed in a subglandular position and she is 41 years old now and has just got pregnant.

Please kindly share your take on this question that the patient posed to me.

E.C.R. Lee, M.B. B.S.

KPJ Sabah Specialist Hospital, No. 2 off Jalan Damai, Luyang,

88300 Kota Kinabalu, Sabah, Malaysia

e-mail: lee.drcharles@gmail.com

D. Topchian, M.B. B.S.

Dr. David \& Associates, Level 1,

Suite 4, 87 Wattletree Road, Armdale,

VIC 3143, Australia

e-mail: david@topchian.com.au

J. Petro, M.D. $(\bowtie)$

Department of Plastic Surgery, New York Medical

College, 40 Sunshine Cottage Rd, Valhalla,

NY 10595, USA

e-mail: DrJPetro@aol.com

\section{Topchian}

If the implant is submammary or subfascial (and especially subpectoral), then there should not be any effect on breast glandular tissue, so breastfeeding should be unaffected.

\section{Petro}

There have been several published studies examining the issue of breastfeeding after augmentation. In some of them, silicone was shown to be present in the breast milk, and this was initially attributed to "leaching" from the implants. Subsequently, it was shown that this was more likely a result of environment absorption or the processing of the milk itself for analysis. Breast implant opponents used the information about silicone in breast milk to make the claim that systemic lupus erythematosus (SLE) was being caused in infants, as well as esophageal motility problems. Again, this has not been confirmed in subsequent studies. It did, however, result in several very large liability settlements. There is no disruption of the duct system with placement of the implant, except when the periareolar approach is used. So unless the implant is inserted in a way that disrupts the ducts, it should have no effect on breastfeeding.

I am enjoying this discussion group and appreciate everyone's willingness to share, so I am thrilled to finally have something to say, too. 